\title{
An Optimization Study of Polyacrylamide- Polyethylenimine-Based Polymer Gel for High Temperature Reservoir Conformance Control
}

\author{
Zulhelmi Amir $\mathbb{D}^{1,},{ }^{1,2}$ Ismail Mohd Saaid $\mathbb{D}^{1},{ }^{1}$ and Badrul Mohamed Jan $\mathbb{D}^{2,3,4}$ \\ ${ }^{1}$ Petroleum Engineering Department, Universiti Teknologi PETRONAS, 32610 Bandar Seri Iskandar, Perak Darul Ridzuan, Malaysia \\ ${ }^{2}$ Department of Chemical Engineering, Faculty of Engineering, University of Malaya, 50603 Kuala Lumpur, Malaysia \\ ${ }^{3}$ Center Advanced Frontier Science, Faculty of Science, University of Malaya, 50603 Kuala Lumpur, Malaysia \\ ${ }^{4}$ Center for Energy Science, Department of Mechanical Engineering, University of Malaya, 50603 Kuala Lumpur, Malaysia
}

Correspondence should be addressed to Ismail Mohd Saaid; ismailsaaid@utp.edu.my

Received 6 July 2018; Accepted 8 August 2018; Published 2 October 2018

Academic Editor: Shu Seki

Copyright ( 92018 Zulhelmi Amir et al. This is an open access article distributed under the Creative Commons Attribution License, which permits unrestricted use, distribution, and reproduction in any medium, provided the original work is properly cited.

\begin{abstract}
This paper presents optimization formulation of organically crosslinked polymer gel for high temperature reservoir conformance control using response surface methodology (RSM). It is always desirable to approach an optimal polymer gel formulation study with adequate performance information related to viscosity and gelation time to minimize excessive water production. In this paper, the effects of polymer and crosslinker concentrations and their influences on gelation time and viscosity were investigated. Central composite design (CCD) was used to determine the optimized organically crosslinked polymer gel formulation. Concentrations of two main raw materials, namely, polyacrylamide (PAM) and polyethylenimine (PEI), were varied in a suitable range. This was to obtain the formulation with the desirable two vital responses, which are viscosity and gelation time. It was found that the results fitted the quadratic equation. Statistically, the quadratic model is reliable and adequate perfectly the variability of the responses obtained from the experimental data. In addition, gelation time and gel viscosity may be controlled by adjusting both polymer and crosslinker concentrations. The optimum formulated organically crosslinked polymer gel with significant desirability factor conditions was achieved at $1.5 \% w / v$ of PAM and $0.3 \% v / v$ of PEI.
\end{abstract}

\section{Introduction}

The well conformance issue is always associated with poor sweep efficiency during waterflooding [1]. Excessive water production is an indication of low sweep efficiency due to poor well conformance [2]. Generally, a perfect well conformance exhibits a consistent sweep across the whole reservoir, while an imperfect well conformance results in a pocket unrecovered oil left behind [3]. There is no reservoir with ideal conformance in the course of its oil recovery process. Heterogeneous or layered oil reservoir formation with high permeability streak is the common nature of a well with conformance challenges. In this case, injected water flows to a wellbore through its easiest own path in the regions with relatively higher permeability. Thus, oil is bypassed by injected water and leads to lots of unswept oil in the relatively lower permeability region. In many mature reservoirs with long waterflooding history, water cuts can easily rise above $90 \%$, greatly reducing the field profitability $[4,5]$. They also lead to active interference in well productivity, early abandonment of the well due to unrecoverable hydrocarbon for mature oilfields, and increase in operating costs to challenge the effect of high produced water $[6,7]$.

Essential treatments have been introduced to minimize excessive water production to increase hydrocarbon production [8]. As one of the various water management strategies, a polymer gel system has become one of the most pertinent blocking agents to mitigate excessive water production [9-12]. Polymer gel can be deployed as a plug in reducing permeability of the high permeability region. Through the altered permeability, a new batch of injected water may change the direction of the flow, which 
displaces fluids into unswept "higher permeability" zones where oil is located. It is the least resistance pathways between the injected water source and production wells during the recovery process [5]. Polymer gel is not limited in improving oil recovery, but it also has other beneficial traits over other methods, such as controllable gelation time, adjustable gel strength, the flexibility for pumping without a work-over rig, in-depth penetration, long effective time, low cost, and easy to remove from the wellbore by water recirculation [11,13-15]. Sorbie and Seright have expounded the concept of gel placement into reservoirs at three stages: injection of low viscosity gelant into the target zones, water injection to displace the low viscosity gel away from the wellbore, and finally shut in well to allow gelation to occur [16]. These polymer gels were designed to propagate through fractures and layers in high permeability and high water saturation zones without damaging productive zones. They help in creating a barrier to prevent water penetration and consequently to reduce the flow of unwanted water in the reservoir $[4,17]$.

A typical gel system usually composed of two main components, namely, high molecular weight polymer and crosslinker. The crosslinking agent starts to connect itself chemically to two polymer molecules and linking them together by internal or external triggers. Nowadays, more reservoirs with high temperatures are being explored and developed, when exploration of new petroleum fields continues [18]. For elevated temperature application, organically crosslinked polymer gel is preferred over metallically crosslinked because of its thermal stability $[17,19-23]$. The covalent bonding between polymer and organic crosslinker provides stable and stronger gels. They have longer gelation times and high thermal stability at high temperatures than coordinated ionic bonding in inorganically crosslinked gel $[17,24]$. The most popular commercial gel used in water management is prepared by polyacrylamide (PAM). PAM is readily available with relatively low costs, and it can deliver desirable viscosity and gel strength to block the high permeability fractures or channels. Meanwhile, polyethylenimine (PEI) is commonly studied organic crosslinker and applied in oilfields worldwide in recent years [25]. This paper presents a study on the application of response surface methodology (RSM) in the optimization of the formulation of polymer gel using middle range molecular weight of PAM crosslinked with PEI. The main objective is to obtain the best gel formulation with maximum achievable gelation time and acceptable viscosity.

\section{Experimental Approach}

2.1. Materials. The used polymer in this study is nonionic polyacrylamide (PAM) that was obtained from SigmaAldrich. It is in powder form with a $\mathrm{pH}$ of 6 and was used as provided. The molecular weight of PAM is with average 5,000,000-6,000,000 mol. wt. Polyethylenimine (PEI) was used as crosslinker provided by BASF with a $\mathrm{pH}$ of approximately 11.7. The molecular weight and active content of PEI were 35,000 mol. wt. and 99\%, respectively, as

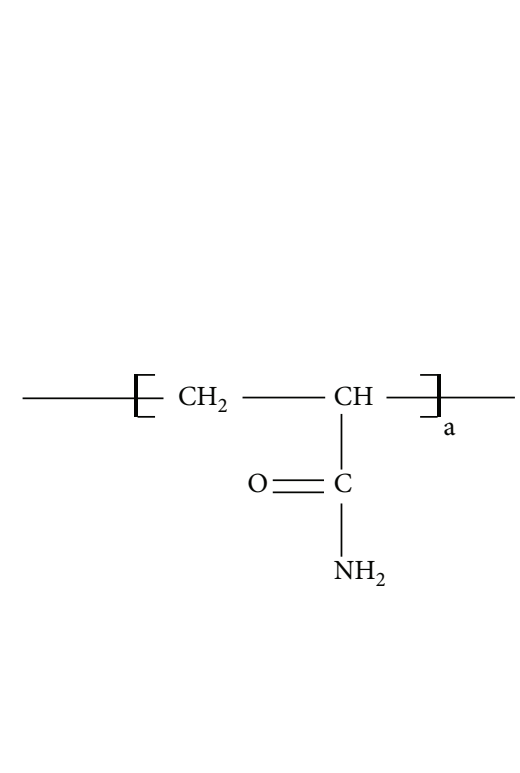

(a)

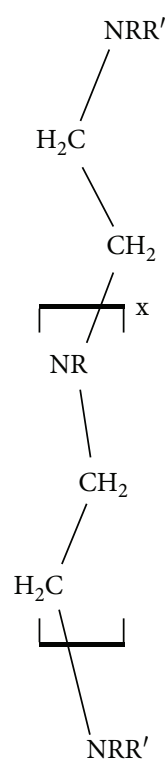

(b)
FIgURE 1: Chemical structure of (a) polyacrylamide and (b) polyethylenimine. Figures from El-Karsani et al. [17].

disclosed by the supplier. The chemical structure of PAM and PEI was given in Figure 1. Deionized water was made in our laboratory.

2.2. Preparation of Polymer Gelant. Polymer gelant in this study was prepared at room temperature. All of the PAM were measured by weight, while PEI were determined by volume. PAM solutions at various concentrations were prepared by mixing initially the polymer powder and deionized water for an hour using the IKA-RW20 digital mixer at $200 \mathrm{rpm}$ to ensure the uniform dissolution of polymer particles. The time and speed of sample mixing were chosen to minimize the shear degradation in PAM samples. Then, various concentrations of PEI were added slowly to the polymer solution. Sequently, hydrochloric acid $(\mathrm{HCl})$ was used to adjust the $\mathrm{pH}$ of the gelling solutions to 7 because the gelation process is optimum in neutral condition. The mixture was then stirred further for another 10 minutes.

2.3. Experimental Design and Data Analysis. Statistical design of the experiments with response surface methodology (RSM) was used to formulate the polymer gel systems with desired performance. The RSM is a useful statistical and mathematical method in modelling and optimization studies. To achieve this objective, the statistical software Design Expert version 11.0.3 (Stat-Ease Inc., Minneapolis, USA) was used. In the first step, the steady shear viscosity measurements were designed by central composite design (CCD) with two factors in five levels. The factors are concentrations of PAM and PEI, while the responses are viscosity and gelation time. The factor column in Table 1 presents the range and combination of the independent variable runs of the CCD approach. The experimental design comprises of four factorial points, four axial points at a distance of \pm 2 from the center, and five replicates of the center point. The two 
TABLE 1: Results of the responses of each experimental run.

\begin{tabular}{lcccc}
\hline Run & \multicolumn{2}{c}{ Factors } & $\begin{array}{c}\text { Responses } \\
\text { Gelation time } \\
\text { (minutes) }\end{array}$ & $\begin{array}{c}\text { Viscosity } \\
\text { (mPa.s) }\end{array}$ \\
\hline 1 & $A$ & $B$ & 56.5 & 478.7 \\
2 & 2.00 & 0.5 & 58.0 & 504.7 \\
3 & 2.00 & 0.5 & 66.5 & 533.5 \\
4 & 2.00 & 0.2 & 75.5 & 286.0 \\
5 & 1.50 & 0.3 & 61.5 & 487.9 \\
6 & 2.00 & 0.5 & 37.5 & 947.6 \\
7 & 3.00 & 0.5 & 59.0 & 531.6 \\
8 & 2.00 & 0.5 & 53.5 & 470.3 \\
9 & 2.00 & 0.5 & 57.0 & 733.4 \\
10 & 2.50 & 0.3 & 41.0 & 620.7 \\
11 & 1.00 & 0.9 & 65.5 & 88.9 \\
12 & 1.50 & 0.7 & 69.5 & 346.5 \\
13 & 2.50 & 0.7 & 45.5 & 736.3 \\
\hline
\end{tabular}

variables were set at five levels which are $-2,-1,0,1$, and 2 . The experiments were conducted as per designed plan before the obtained data were plugged into the software. The CCD approach was employed to generate mathematical models from experimental data. The correlation can be used to describe comprehensive relationship between the factors and responses and to predict the gelation time and viscosity of the polymer gel. Furthermore, three-dimensional plots and their respective contour plots were also considered to analyze the relationship. The correlation in CCD is usually shown as a quadratic equation. Then, analysis of variance (ANOVA) was used to analyze statistically the effects of process variables, which is to evaluate the reliability and accuracy of the generated model. To confirm the reliability of the generated models, the $R^{2}$ value was calculated. Then, its statistical significance was determined by Fisher's $F$-test using the same program. In addition, $P$ value (probability of error) with $95 \%$ confidence level was used to evaluate the model terms. Finally, in the optimization process, the formulation for polymer gelant was established based on the maximum possible gelation time and acceptable minimum viscosity.

2.4. Viscosity and Gelation Time Measurements. Literature reported some methods applied to determine the gelation kinetics $[19,26,27]$. The methods that are usually used include the bottle test, the sealed tube, the dynamic shear using rheometer, and the static shear using viscometer methods. In this study, the static shear method was used to evaluate the effects of different concentrations of raw materials on the gelation time and viscosity of polymer gel. The steady shear method has been widely used because this technique provides a quantitative measurement of gelation rate, gelation time, and gel viscosity. Moreover, this method is reasonably faster to get the results, accurate, and not very costly compared than the other methods. It has been used by several researchers to determine the gelation time $[19,28]$. The viscosity and gelation time were determined from the curves of viscosity against time at high temperatures. The inflection point of the viscosity versus time curve was considered as the gelation point. In the present study, the viscosity and gelation time measurements were taken using a rotational Brookfield Viscometer Model DV-II (AMETEK Brookfield, Massachusetts, USA). This viscometer is equipped with a cylindrical sample holder, a heating jacket, a spindle, and a sealing element. During each test, the gelling solutions were prepared and placed into the sample holder that has been preheated to desired temperature. The volume of the sample was approximately $8 \mathrm{ml}$. The measurements were taken at temperature of $95^{\circ} \mathrm{C}$ and ambient pressure. The temperature was controlled by a thermostatic oil bath (Haake $\mathrm{CH}$ bath reservoir with Haake F3 temperature controller) connected to the viscometer. Then, the spindle was immersed into the sample, and a steady shear rate of $5.0 \mathrm{~s}^{-1}$ was applied for all experiments. The sample was always stirred by the viscometer spindle in the sample cup throughout the experiment. The condition to ensure the temperature throughout the sample was the same. Viscosity data was automatically worked out using a software, and the desired viscosity data was recorded in correspondence to experimental time. Based on the CCD designed matrix, all measuring runs were performed randomly to minimize the effect of unexpected variability in the observed responses. Finally, laboratory tests were carried out in the same conditions based on the generated optimal formulation. This is to validate the mathematical values generated by the software. The error and accuracy between the predicted and experimental values were obtained to determine the precision of the software-generated formula.

\section{Results and Discussions}

3.1. Gelation Kinetics and Statistical ANOVA Analysis. Measurements of PAM/PEI polymer gel viscosities were performed at $95^{\circ} \mathrm{C}$. Figure 2 shows the relationship of $\mathrm{PAM} / \mathrm{PEI}$ polymer gel viscosities as a function of experimental time. These results have been used for the ANOVA statistical analysis as presented in Table 1. The CCD design in RSM was used to investigate the correlation between the two independent variables, namely, concentrations of polyacrylamide and polyethyleimine, and the two responses, namely, viscosity and gelation time. The analysis predicted and generated mathematical models for each of the responses. These results were used to assess the predicted responses for viscosity and gelation time as a function of polyacrylamide and polyethylenimine concentrations.

3.1.1. Gelation Time. The experimental results as shown in contour and 3D response surface plots (Figure 3) indicate the relationship between gelation time, as a function of polyacrylamide and polyethylenimine concentrations. The gelation time is crucial because it is used in oilfield applications to determine the ability of PAM/PEI polymer gelant to travel deeper through the reservoir. The injection time and depth of propagation of the gel solution into the formation are a function of gelation time. There are different definitions of gelation time reported. Firstly, it is described as the time required to achieve a specific viscosity [29]. Secondly, it is the time required to achieve a specific gel strength [30]. In 


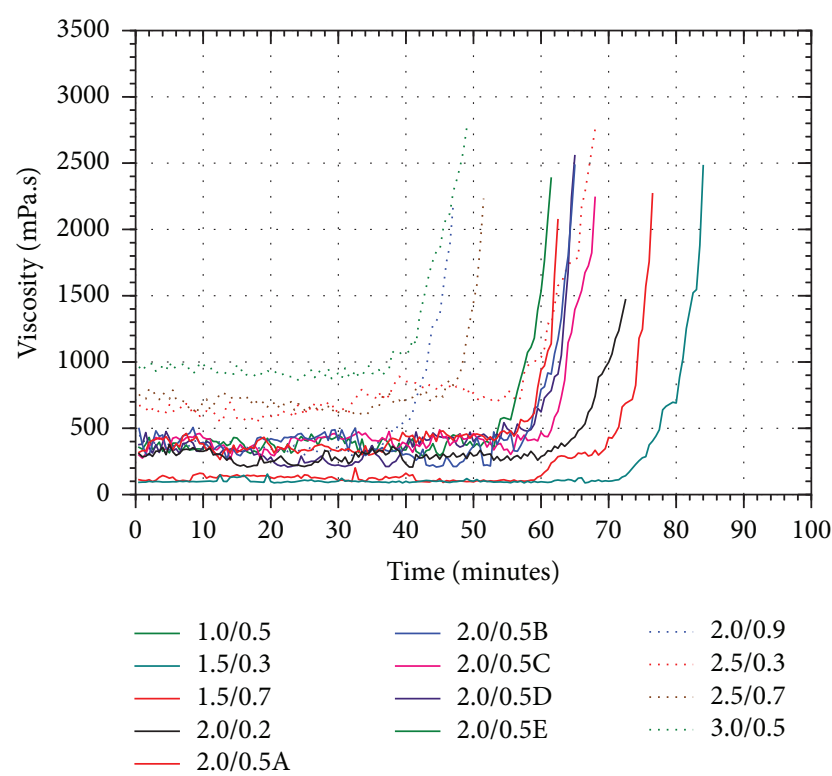

FIgURe 2: Viscosity of PAM/PEI polymer gel against experimental time at various polymer and crosslinker concentrations.

this case, oscillatory viscometer is commonly used to determine the gelation time by measuring the changes of gel strength as a function of time. The maximum slope in the curve of elastic modulus against time can be taken as the gelation period [31]. The last definition of the gelation time is the time required for the elastic and viscous moduli to intersect, and the moduli ratio becomes independent of frequency [32]. In this study, the gelation time is defined as the duration of time required for viscosity to reach the inflection point on the curve of viscosity versus time [21]. The inflection point in the gelation process can be observed as rapidly increasing in viscosity after it starts slowly with no apparent viscosity increment at the beginning. This definition has been used by numerous researches to define the gelation time $[26,28]$.

It can be seen in Figure 3 that as the polymer concentration increases at the same crosslinker concentration, the gelation time decreases. For instance, when the polymer concentration was less than $2.0 \%$, the gelation time was longer than 60 minutes. Conversely, when the polymer concentration was higher than $3.0 \%$, the gelation time was shorter than 50 minutes. As the polymer concentration or the number of crosslinking sites increases, the rate of gel formation increases, and thereby, the gelation time increases. Similar to the effect of polymer concentration, the gelation time of polymer gel was found to be fairly affected by the initial crosslinker concentrations. Experimental results showed that if the crosslinker system concentration was less than $0.5 \%$, the gelation time would be more than 70 minutes. This is expected because higher PEI concentrations offer more crosslinking opportunities for the polymer. This can also be explained that the available imine groups increased with the increase of PEI content; hence, the crosslinking rate is improved, and consequently, the gelation time shortened. The functional groups of PAM, which are amide groups $\left(-\mathrm{CONH}_{2}\right)$, will immediately form gel as soon as they react with crosslinker under high temperature. This observation is in agreement with the data reported for organically crosslinked systems [21]. However, it can be observed that the rate of the increase of gelation time by the effect of PEI is less pronounced compared with the effect of PAM. There is also an understanding that delaying gelation is more efficient by retardation of polymer reaction over the crosslinker side [17]. Therefore, PAM is more dominant over PEI in determining the gelation time of the PAM/PEI gel system.

Experimental results for the first response, which is gelation time, were fitted into the equation by the analysis of variance (ANOVA). This equation is a mathematical model that can be used to predict the desired responses at any of the respective factors. ANOVA was used to obtain the model that fits the most to the results. The best equation that fits the results of gelation time is quadratic in nature. The quadratic model to describe the correlation between gelation time $\left(Y_{1}\right)$ as a function of concentration of raw materials in terms of coded factors is expressed as

$$
Y_{1}=59.41-8.21 A-6.09 B-1.37 A B-1.42 A^{2}-0.56 B^{2},
$$

where $A$ represents polyacrylamide concentration and $B$ represents polyethylenimine concentration.

ANOVA analysis was also used to confirm the accuracy of the obtained model. The results of ANOVA analysis show that the model $F$ value given was 8.74 , which indicates that the model is significant. It gave indication that the model has only $0.64 \%$ chance to occur due to noise. The data also indicates that the mathematical model is significant at $5 \%$ confidence level because the value of probability of error $(P)$ is less than 0.05 . In addition, based on the results, the obtained model gives a very high $R^{2}$ and adjusted $R^{2}$ values of 0.8619 and 0.7633 , respectively. According to Noordin et al., it is necessary to achieve high $R^{2}$ value that is close to 1 [33]. Moreover, having a good agreement with the adjusted $R^{2}$ value is also desirable. The high value of $R^{2}$ and adjusted $R^{2}$ validates the quadratic model is appropriate and satisfactorily fit to the variability of the responses produced from experimental data. The lack of fit $F$ value is 6.31. Even though the lack of fit value relatively has low probability of $5.37 \%$ chance to occur due to noise, the lack of fit value implies that it is not significant relative to the pure error. A nonsignificant lack of fit is required as it demonstrates that the model is reliable.

In addition, from the ANOVA results, it is possible to evaluate the signal to noise ratio of the experimental data by assessing the adequate precision (AP). AP value compares the range of the predicted value at the design points [34]. A ratio of more than 4 is anticipated since this value shows the model discrimination. It means the model can be applied to navigate the design space [35]. Based on the results, the response for gelation yielded a high AP value of 9.0485, which is more than 4 . This indicates that the predicted model provides an excellent ability to navigate the design space 


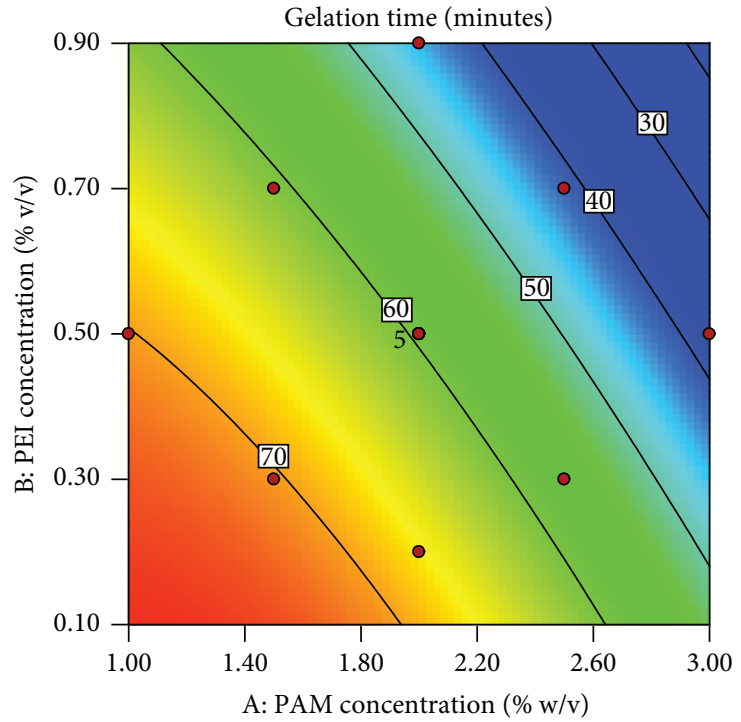

Design-Expert ${ }^{\circledR}$ Software

Factor coding: actual

Gelation time (minutes)

- Design points

$37.5 \square 75.5$

$\mathrm{X} 1=\mathrm{A}: \mathrm{PAM}$ concentration

$\mathrm{X} 2=\mathrm{B}$ : PEI concentration

(a)

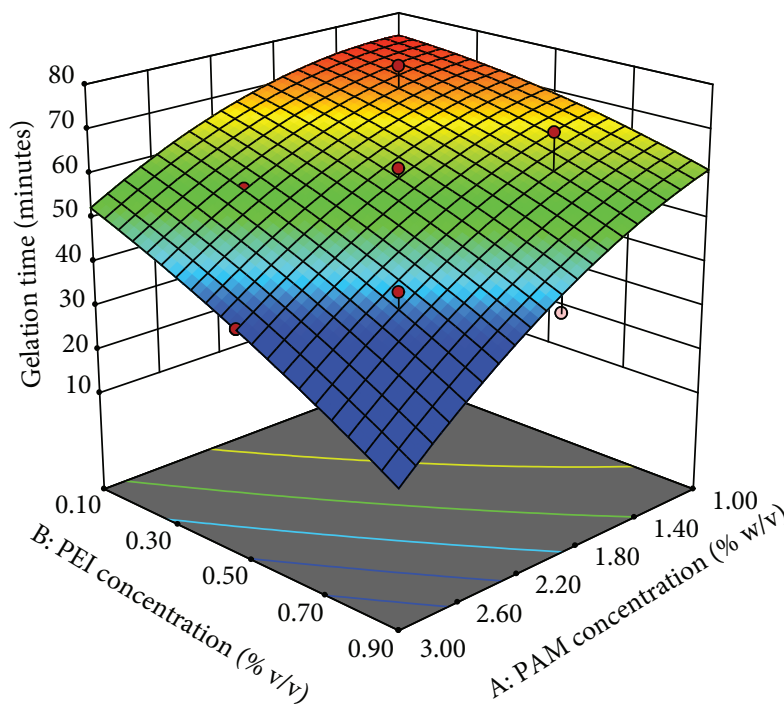

Design-Expert ${ }^{\oplus}$ Software

Factor coding: actual

Gelation time (minutes)

- Design points above predicted value

- Design points below predicted value

$37.5 \square 75.5$

$\mathrm{X} 1=\mathrm{A}: \mathrm{PAM}$ concentration $\mathrm{X} 2=\mathrm{B}$ : PEI concentration

(b)

Figure 3: Contour and 3D response surface plots from Design Expert for gelation time versus concentration of materials.

designed by the CCD. This is supported by a comparison plot between the predicted and actual values. This comparison plot confirms the accuracy and reliability of the model. Figure 4 presents the diagnostic graph between the predicted and actual values for gelation time. The meaning of the plots is described by the position of the points. Points that are lying above the diagonal line indicate that the values were overestimated, while those below the diagonal line indicate underestimated values [36]. It can be observed that all points are distributed along the diagonal line. The predicted values are in adequate agreement with the actual experimental values. Hence, it could be suggested that the obtained model gives a satisfactory estimation of the predicted values and may be used in predicting the gelation time.

3.1.2. Viscosity. The second response in this study is viscosity of the PAM/PEI based polymer gel at the gelation point. Viscosity is a crucial property of the liquid that manifests the relative motion of the molecules [37]. Basically, liquid flows until stress has diminished and energy has dissipated through the friction. Contradict to the trend of gelation time, the increment of all of the raw material concentrations tend to cause the viscosity of the polymer gel to increase. Viscosity can be influenced by the increment of polymer concentration as can be seen in Figure 5. Based on the results, when the polymer concentration was higher than $2.0 \%$, the viscosity was higher than $400 \mathrm{mPa}$.s. Similarly, when the polymer concentration was almost $2.5 \%$, the viscosity is more than $800 \mathrm{mPa}$.s. This is due to the higher polymer concentration that promotes the thickening process in the solutions. The thickening capability of PAM originated from the high molecular weight. In addition, as the polymer loading increase, more polymer chain could stretch in the aqueous solution. The repulsion of the negative electrostatic charges on the polymer chains is the main reason of polymeric stretching in aqueous solution, which consequently causes viscosity to increase $[38,39]$. As a result, more crosslinkable sites will be accessible on the polymer backbone, consequently created a denser network of polymer [40]. Moreover, a higher number of polymer molecules lead to higher interactions. Thus, it leads to molecular entanglements between polymer chains [41]. This phenomenon is the reason that upholds higher viscosity for the polymer solutions [39]. On the other hand, the viscosity was found not affected significantly with the increase of crosslinker concentration. Based on the experimental results, polymer concentration seems to have much more influence over crosslinker concentration in determining the viscosity of the gel system [42]. It seems to suggest that a production of lower viscosity of polymer system required lower loading of polymer and crosslinker.

The results from the experimental works were used for the model fitting by ANOVA. Similar to the first response, the quadratic mathematical model is the most suitable for viscosity. The quadratic model to represent the relationship 


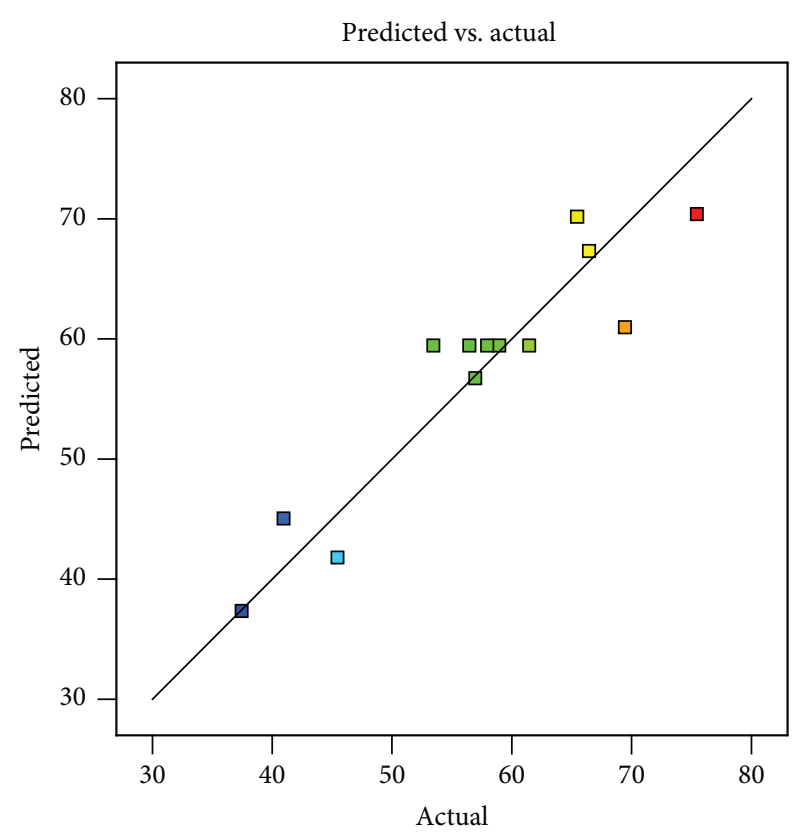

Design-Expert ${ }^{\circ}$ Software

Gelation time

Color points by value of

gelation time:

$37.5 \square 75.5$

FIGURE 4: Predicted versus actual values plot from Design Expert for gelation time of PAM/PEI polymer gel.

between concentration of raw materials and viscosity $\left(Y_{2}\right)$ in terms of coded factors is as follows

$$
Y_{2}=494.88+212.88 A+13.82 B-14.4 A B+5.83 A^{2}+24.95 B^{2},
$$

where $A$ represents polyacrylamide concentration and $B$ represents polyethylenimine concentration.

From the results of ANOVA analysis, the model $F$ value is 317.51 , which means that the model is significant. The probability or $P$ value is established to be lower than 0.05 , which implies that the model terms, which are $A$ and $A^{2}$ are significant. Values greater than 0.1 indicate the model terms are not significant. The $R^{2}$ and adjusted $R^{2}$ values are also reasonably near to 1.0 , which are 0.9956 and 0.9925 , respectively. The generated mathematical equation is well fitted when the difference between $R^{2}$ and adjusted $R^{2}$ values is less than 0.2. The lack of fit $F$ value of 0.06 indicates that the lack of fit is not significant relative to the pure error. For a lack of fit value this large to occur due to noise, it needs a chance of $97.8 \%$. As mentioned before, nonsignificant lack of fit is desired so that the model can be used to represent the viscosity. The AP ratio obtained in this analysis was 66.78 which was greater than 4 . This is desirable and signifies that the model is reliable to navigate the design space. A comparison plot between the predicted and actual values has also been referred to justify the accuracy of the model. Figure 6 illustrates the statistical comparison plot between experimental values and the predicted values for viscosity using the ANOVA generated model. Based on the plot, it is clear that the actual values for gelation time are considered well fitted to predicted values with acceptable variance range. As can be seen, all the experiment design points are lying almost at the same spot and close to the straight line. This suggests that the generated models may provide a good viscosity prediction for PAM/PEI polymer gel.

3.2. Optimization of Polymer Gel Formulation. During the treatment of a deep reservoir, it is important to consider both the effect of gelation time and gelant viscosity when designing the required amount of polymer and crosslinker loading. It should be noted that optimum value of polymer and crosslinker concentration need to be determined. For example, the addition of high crosslinker may cause syneresis [43, 44]. Syneresis must be avoided, especially when gel is employed in naturally fractured reservoirs. Syneresis is caused by overcrosslinking from excessive concentration of crosslinker. Consequently, the water will expulse out of the gel by osmosis and polymer gel dehydrate [45]. Because of this, gel volume reduces, and new flow paths are created, eventually affecting the performances of gel treatment. Conversely, with increasing the polymer dosage, the time required to get a nonflowing polymer gel with a tolerable strength will be shortened. The main objective of this optimization part is to determine the optimum concentrations of raw materials. It supposedly gives the optimized formulation for PAM/PEI polymer gel that is reliable to be applied for high temperature reservoir conformance control. During the optimization process, the polymer gel is setting to have the acceptable lowest viscosity and possible maximum gelation time. A low to very low viscosity of the formulated polymer gel is desirable to obtain good carrying capacity of the fluid and longer propagation of gelant through the reservoir matrix. On the other hand, maximum gelation time is needed for longer propagation and to avoid the gel forming inside the wellbore. Thus, in order to simultaneously optimize all the two factors (concentrations of polyacrylamide and polyethylenimine) correlated to the responses (gelation time and viscosity), a desirability function $(d)$ for multiple responses was used [46]. The values were generated by the Design Expert software when performing the multiple response optimization based on

$$
d=\left[\prod_{i=1}^{N} m_{i}^{r_{i}}\right]^{1 / \sum r_{i}},
$$

where $N$ represents the number of responses, $r_{i}$ indicates the importance of particular response, and $m_{i}$ is the partial desirability function for specific responses.

Generally, the highest value of desirability $d$ represents the optimum conditions that can be achieved from the optimization process [46]. In the optimization process, the gelation time of the PAM/PEI polymer gel was set more important than viscosity. Based on the results, the highest desirability value is 0.898 . At this $d$ value, the values of the 

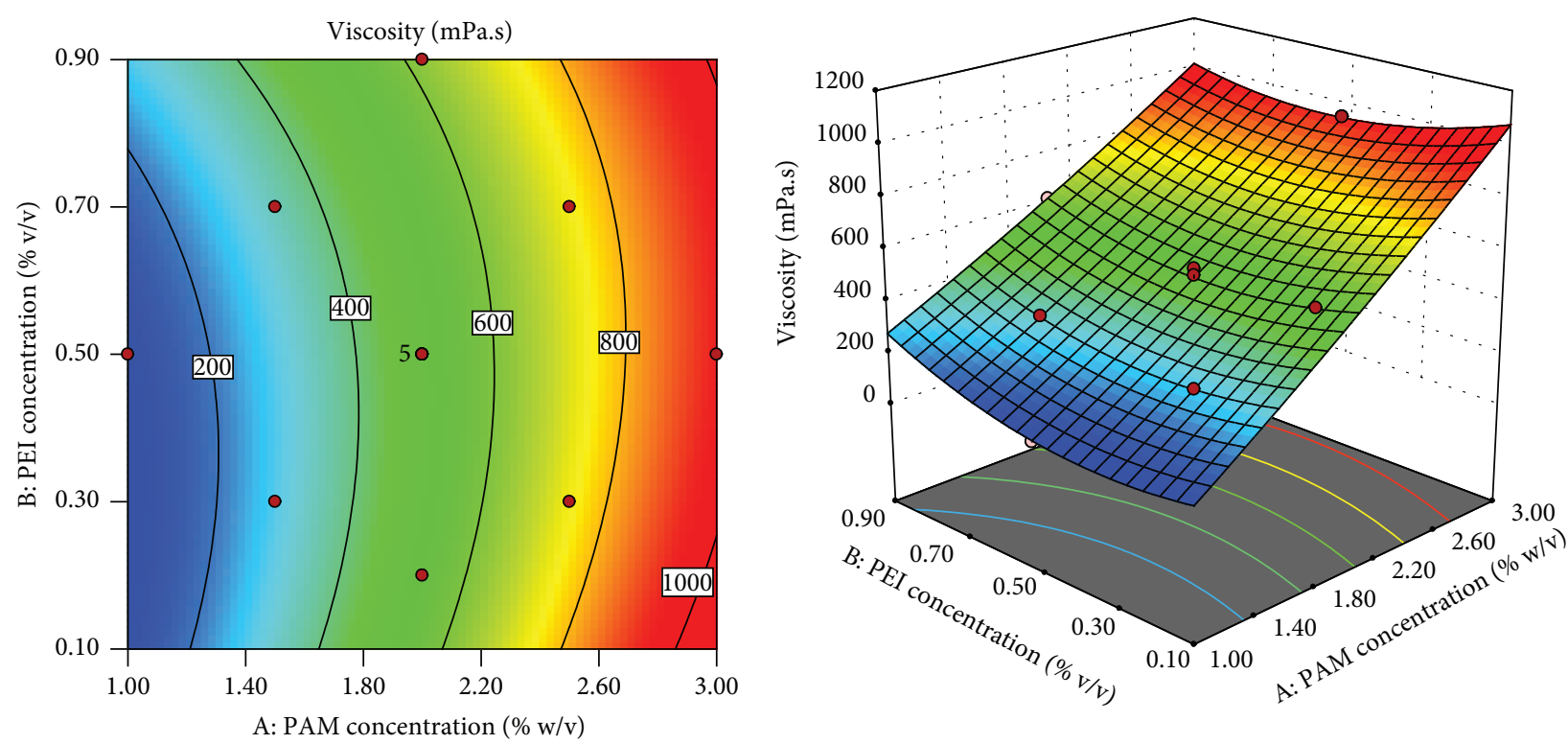

Design-Expert ${ }^{\circledR}$ Software

$$
\begin{aligned}
& \text { Factor coding: actual } \\
& \text { Viscosity (mPa.s) } \\
& \text { ○ Design points } \\
& 88.9 \\
& 947.582
\end{aligned}
$$

$\mathrm{X} 1=\mathrm{A}: \mathrm{PAM}$ concentration

$\mathrm{X} 2$ = B: PEI concentration

(a)

$$
\begin{aligned}
& \text { Design-Expert }{ }^{\circledR} \text { Software } \\
& \text { Factor coding: actual } \\
& \text { Viscosity (mPa.s) } \\
& \text { ○ Design points above predicted value } \\
& \text { O Design points below predicted value } \\
& 88.9 \square 947.582
\end{aligned}
$$

$\mathrm{X} 1=\mathrm{A}: \mathrm{PAM}$ concentration $\mathrm{X} 2=\mathrm{B}: \mathrm{PEI}$ concentration

(b)

FIGURE 5: Contour and 3D response surface plots from Design Expert for viscosity versus concentration of materials.

partial desirability, $m_{i}$, for the two responses, gelation time and viscosity, are 0.86 and 0.23 , respectively. This value corresponds to the optimum formulation where the value of factor $A$ (concentration of polyacrylamide) is $1.5 \% \mathrm{w} / \mathrm{v}$ and factor $B$ (concentration of polyethylenimine) is $0.3 \% v / v$. The predicted values for each response parameters, which are gelation time viscosity $\left(Y_{1}\right)$ and viscosity $\left(Y_{2}\right)$, are 70.3 minutes and $284.6 \mathrm{mPa}$.s, respectively. According to AlMuntasheri et al. [47], a good range of gelation time for polymer gel should be more than 55 minutes. Since the gelation time for the optimized formulation is about 70 minutes, it is considered good as it is well above 55 minutes. The viscosity on the beginning of the gelation point is relatively within the acceptable range for in situ polymer gel. Then, the $d$ value is the highest achievable desirability; it could considerably represent the optimum condition for the process optimization. Thus, this condition was selected as the optimum concentration of polyacrylamide and polyethylenimine to formulate the PAM/PEI polymer gel.

To validate the optimum process conditions acquired from response surface methodology using CCD, a laboratory experimental test was conducted based on selected optimum conditions. Predicted and experimental conditions were maintained the same for a fair comparison between the two. The test was conducted by formulating the PAM/PEI polymer gel using the optimized formulations as mentioned above. The corresponding gelation time and viscosity were measured as shown in Figure 7 and then was compared to the predicted response values in optimum process condition calculated from the mathematical model. Based on the results, it can be concluded that the two response parameters obtained from the experiment are in good agreement with predicted values. This is due to the high value of calculated accuracy for gelation time and viscosity, which are $97 \%$ and $86.5 \%$, respectively. Value of error is also low, which are 2.2 and 38.4 for gelation time and viscosity, respectively. In addition, low value of standard deviation $(\sigma)$ is shown with values of 1.1 and 19.2 for gelation and viscosity.

\section{Conclusions}

A polymer gel incorporating polyacrylamide and polyethylenimine has been successfully formulated with the desired properties from RSM. The response surface plots showed that the viscosity of the gelant increases with increasing material concentrations. Gelant viscosity tends to increase with polyacrylamide and polyetylenimine concentrations. Gelation time of the PAM/PEI-based gel also tend to decrease with increasing material concentration. The experimental results for all responses fit the quadratic mathematical model predictions. For gelation time and viscosity, polymer concentration affects these two responses the most. It is concluded that the optimum concentrations for the 


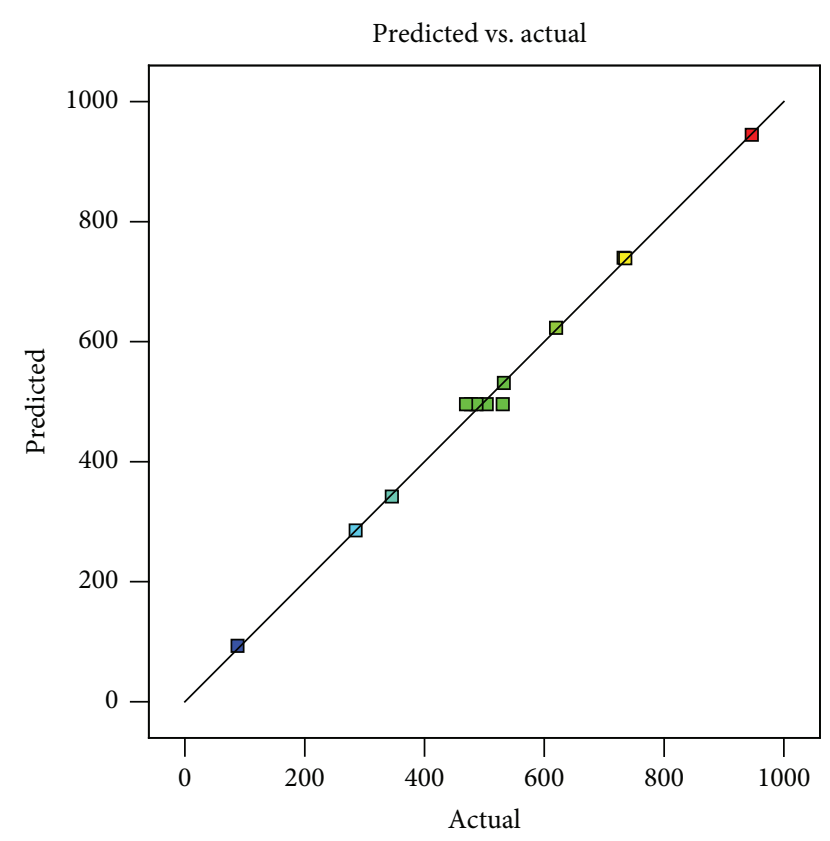

Design-Expert ${ }^{\oplus}$ Software

Viscosity

Color points by value of viscosity:

88.9 ПII 947.582

Figure 6: Predicted versus actual values plot from Design Expert for viscosity of PAM/PEI polymer gel.

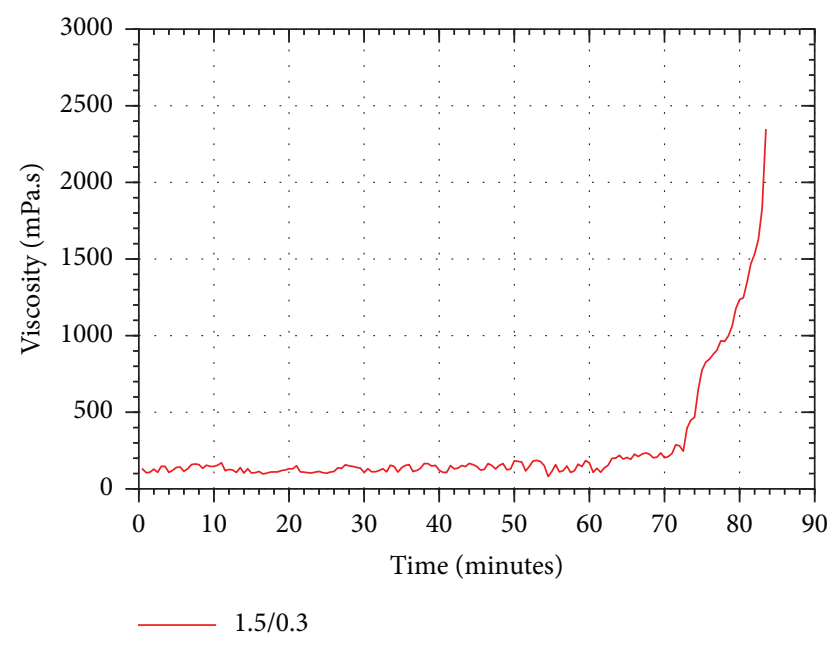

FIgURe 7: Viscosity of PAM/PEI polymer gel against experimental time at optimized polymer and crosslinker concentration.

formulation is achieved at the following conditions where the value of factor $A$ (concentration of polyacrylamide) is $1.5 \%$ $w / v$, and factor $B$ (concentration of polyethylenimine) is $0.3 \% v / v$. This formulation has gelation time of 70.4 minutes and viscosity of $284.6 \mathrm{mPa}$.s. Furthermore, from the validation experiment, it could be concluded that the two response parameters obtained from both the experiments and predicted by the models were in a good agreement.

\section{Data Availability}

The Design Expert data used to support the findings of this study are available from the corresponding author upon request.

\section{Conflicts of Interest}

The authors declare that they have no conflict of interest.

\section{Acknowledgments}

The authors appreciate the contributions from Universiti Teknologi Petronas (YUTP 0153AA-H05), PETRONAS (GR\&T 0153CB-019), University of Malaya (UMRG RP031B-15AFR), and SLAI Fellowship Scheme from Ministry of Education Malaysia and University of Malaya.

\section{References}

[1] R. D. Sydansk and L. Romero-Zeron, Reservoir Conformance Improvement, Society of Petroleum Engineers Richardson, TX, 2011.

[2] B. Bailey, M. Crabtree, J. Tyrie et al., "Water control," Oilfield Review, vol. 12, pp. 30-51, 2000.

[3] D. Borling, K. Chan, T. Hughes, and R. Sydansk, "Pushing out the oil with conformance control," Oilfield Review, vol. 6, pp. 44-58, 1994.

[4] R. D. Sydansk and G. P. Southwell, "More than 12 years of experience with a successful conformance-control polymer gel technology," in SPE/AAPG Western Regional Meeting, Long Beach, California, June 2000.

[5] R. D. Sydansk and R. S. Seright, "When and where relative permeability modification water-shutoff treatments can be successfully applied," SPE Production \& Operations, vol. 22, no. 2, pp. 236-247, 2007.

[6] R. L. Hirsch, R. Bezdek, and R. Wendling, "Peaking of world oil production and its mitigation," AICHE Journal, vol. 52, no. 1, pp. $2-8,2006$.

[7] R. S. Seright, R. H. Lane, and R. D. Sydansk, "A strategy for attacking excess water production," SPE Production \& Facilities, vol. 18, no. 3, pp. 158-169, 2003.

[8] R. Seright, "Washout of $\mathrm{Cr}(\mathrm{III})$-acetate-HPAM gels from fractures," in International Symposium on Oilfield Chemistry, Houston, Texas, February 2003.

[9] A. Kabir, "Chemical water \& gas shutoff technology—an overview," in SPE Asia Pacific Improved Oil Recovery Conference, Kuala Lumpur, Malaysia, October 2001.

[10] J.-P. Coste, Y. Liu, B. Bai et al., "In-depth fluid diversion by pre-gelled particles. Laboratory study and pilot testing," in SPE/DOE improved oil recovery symposium, Tulsa, Oklahoma, April 2000.

[11] A. Prada, F. Civan, and E. D. Dalrymple, "Evaluation of gelation systems for conformance control," in SPE/DOE improved oil recovery symposium, Tulsa, Oklahoma, April 2000.

[12] G. A. Al-Muntasheri, H. A. Nasr-El-Din, J. Peters, and P. L. J. Zitha, "Investigation of a high temperature organic water shutoff gel: reaction mechanisms," SPE Journal, vol. 11, no. 4, pp. 497-504, 2006. 
[13] A. Hamouda and H. Amiri, "Factors affecting alkaline sodium silicate gelation for in-depth reservoir profile modification," Energies, vol. 7, no. 2, pp. 568-590, 2014.

[14] H. Akhlaghi Amiri, A. Hamouda, and A. Roostaei, "Sodium silicate behavior in porous media applied for in-depth profile modifications," Energies, vol. 7, no. 4, pp. 2004-2026, 2014.

[15] D. Perez, F. E. Fragachan, A. R. Barrera, and J. P. Feraud, "Applications of polymer gel for establishing zonal isolations and water shutoff in carbonate formations," SPE Drilling \& Completion, vol. 16, no. 3, pp. 182-189, 2001.

[16] K. S. Sorbie and R. S. Seright, "Gel placement in heterogeneous systems with crossflow," in SPE/DOE Enhanced Oil Recovery Symposium, Tulsa, Oklahoma, April 1992.

[17] K. S. M. El-Karsani, G. A. Al-Muntasheri, and I. A. Hussein, "Polymer systems for water shutoff and profile modification: a review over the last decade," SPE Journal, vol. 19, no. 1, pp. 135-149, 2014.

[18] R. Ziegler, "Technology focus: high-pressure/high-temperature challenges (April 2017)," Journal of Petroleum Technology, vol. 69, no. 4, pp. 79-79, 2017.

[19] D. Broseta, O. Marquer, N. Blin, and A. Zaitoun, "Rheological screening of low-molecular-weight polyacrylamide/chromiu$\mathrm{m}$ (III) acetate water shutoff gels," in SPE/DOE improved oil recovery symposium, Tulsa, Oklahoma, April 2000.

[20] A. Moradi-Araghi, "A review of thermally stable gels for fluid diversion in petroleum production," Journal of Petroleum Science and Engineering, vol. 26, no. 1-4, pp. 1-10, 2000.

[21] G. A. Al-Muntasheri, H. A. Nasr-El-Din, and I. A. Hussein, “A rheological investigation of a high temperature organic gel used for water shut-off treatments," Journal of Petroleum Science and Engineering, vol. 59, no. 1-2, pp. 73-83, 2007.

[22] K. S. M. El-Karsani, G. A. Al-Muntasheri, A. S. Sultan, and I. A. Hussein, "Gelation of a water-shutoff gel at high pressure and high temperature: rheological investigation," SPE Journal, vol. 20, no. 5, pp. 1103-1112, 2015.

[23] R. S. Seright and F. D. Martin, "Impact of gelation $\mathrm{pH}$, rock permeability, and lithology on the performance of a monomer-based gel," SPE Reservoir Engineering, vol. 8, no. 1, pp. 43-50, 1993.

[24] J. E. Vasquez, E. D. Dalrymple, I. Abbasy, and L. S. Eoff, "Laboratory evaluation of water swellable materials for fracture shutoff," in SPE North Africa Technical Conference \& Exhibition, Marrakech, Morocco, March 2008.

[25] J. Morgan, P. Smith, and D. Stevens, "Chemical adaptation and development strategies for water and gas shutoff gels," in RSC Chemistry in the Oil Industry, 6th International Symposium, Charllotte Mason College Ambleside, UK, 1997.

[26] J. Vasquez, E. D. Dalrymple, L. Eoff, B. Reddy, and F. Civan, "Development and evaluation of high-temperature conformance polymer systems," in SPE International Symposium on Oilfield Chemistry, The Woodlands, Texas, February 2005.

[27] J. Vasquez, F. Civan, T. M. Shaw et al., "Laboratory evaluation of high-temperature conformance polymer systems," in SPE production and operations symposium, Oklahoma City, Oklahoma, March 2003.

[28] B. R. Reddy, L. Eoff, E. D. Dalrymple, K. Black, D. Brown, and M. Rietjens, "A natural polymer-based cross-linker system for conformance gel systems," SPE Journal, vol. 8, no. 2, pp. 99106, 2003.

[29] D. S. Jordan, D. W. Green, R. E. Terry, and G. P. Willhite, “The effect of temperature on gelation time for polyacrylamide/ chromium (III) systems," Society of Petroleum Engineers Journal, vol. 22, no. 4, pp. 463-471, 1982.

[30] R. D. Sydansk, "Acrylamide-polymer/chromium (III)-carboxylate gels for near wellbore matrix treatments," SPE Advanced Technology Series, vol. 1, no. 1, pp. 146-152, 1993.

[31] R. Prud'homme and J. Uhl, "Kinetics of Polymer/Metal-Ion Gelation," in SPE Enhanced Oil Recovery Symposium, Tulsa, Oklahoma, April 1984.

[32] A. Ponton, S. Warlus, and P. Griesmar, "Rheological study of the sol-gel transition in silica alkoxides," Journal of Colloid and Interface Science, vol. 249, no. 1, pp. 209-216, 2002.

[33] M. Y. Noordin, V. C. Venkatesh, S. Sharif, S. Elting, and A. Abdullah, "Application of response surface methodology in describing the performance of coated carbide tools when turning AISI 1045 steel," Journal of Materials Processing Technology, vol. 145, no. 1, pp. 46-58, 2004.

[34] M. Khalil, B. Mohamed Jan, and A. Aziz Abdul Raman, "Fractional factorial design optimization of nontraditional completion fluid for perforation with underbalance," Chemistry and Technology of Fuels and Oils, vol. 46, no. 5, pp. 340-350, 2010.

[35] Q. K. Beg, V. Sahai, and R. Gupta, "Statistical media optimization and alkaline protease production from Bacillus mojavensis in a bioreactor," Process Biochemistry, vol. 39, no. 2, pp. 203-209, 2003.

[36] A. L. Ahmad, S. C. Low, S. R. A. Shukor, and A. Ismail, "Optimization of membrane performance by thermal-mechanical stretching process using responses surface methodology (RSM)," Separation and Purification Technology, vol. 66, no. 1, pp. 177-186, 2009.

[37] P. C. Hiemenz and T. P. Lodge, Polymer Chemistry, CRC Press, 2007.

[38] R. J. Stokes and D. F. Evans, Fundamentals of Interfacial Engineering, John Wiley \& Sons, 1997.

[39] D. A. Z. Wever, F. Picchioni, and A. A. Broekhuis, "Polymers for enhanced oil recovery: a paradigm for structure-property relationship in aqueous solution," Progress in Polymer Science, vol. 36, no. 11, pp. 1558-1628, 2011.

[40] R. Reichenbach-Klinke, B. Langlotz, B. Wenzke, C. Spindler, and G. Brodt, "Associative copolymer with favorable properties for the application in polymer flooding," in SPE International Symposium on Oilfield Chemistry, The Woodlands, Texas, USA, April 2011.

[41] R. P. Wool, "Polymer entanglements," Macromolecules, vol. 26, no. 7, pp. 1564-1569, 1993.

[42] K. S. M. ElKarsani, G. A. al-Muntasheri, A. S. Sultan, and I. A. Hussein, "Performance of PAM/PEI gel system for water shut-off in high temperature reservoirs: laboratory study," Journal of Applied Polymer Science, vol. 132, no. 17, 2015.

[43] S. L. Bryant, M. R. Rabaioli, and T. P. Lockhart, "Influence of syneresis on permeability reduction by polymer gels," SPE Production \& Facilities, vol. 11, no. 4, pp. 209-215, 1996.

[44] R. W. Eggert Jr, G. P. Willhite, and D. W. Green, "Experimental measurement of the persistence of permeability reduction in porous media treated with xanthan/Cr(III) gel systems," SPE Reservoir Engineering, vol. 7, no. 1, pp. 29-35, 1992.

[45] Q. Ren, H. Jia, D. Yu et al., "New insights into phenol-formaldehyde-based gel systems with ammonium salt for low-temperature reservoirs," Journal of Applied Polymer Science, vol. 131, no. 16, 2014. 
[46] D. Montgomery, Design and Analysis of Experiments Fifth Edition, By John Wiley \& Sons, Inc. All rights reserved, 2001.

[47] G. A. Al-Muntasheri, L. Sierra, F. O. Garzon, J. D. Lynn, and G. A. Izquierdo, "Water shut-off with polymer gels in a high temperature horizontal gas well: a success story," in SPE Improved Oil Recovery Symposium, Tulsa, Oklahoma, USA, April 2010. 


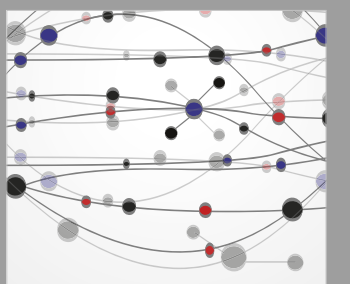

The Scientific World Journal
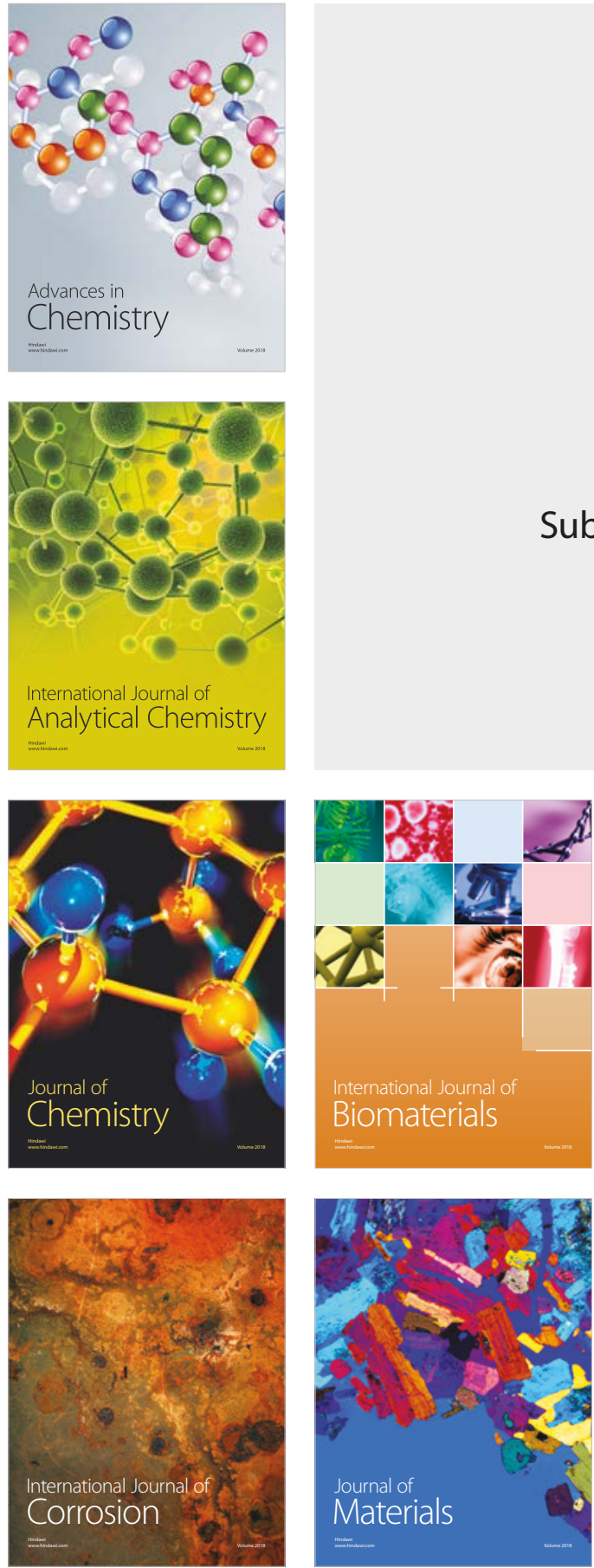

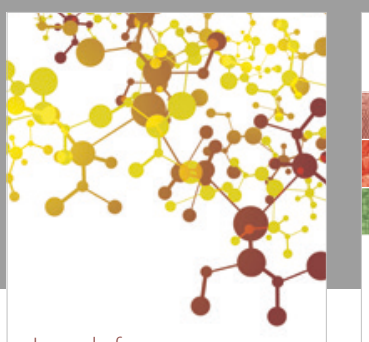

Journal of

Applied Chemistry
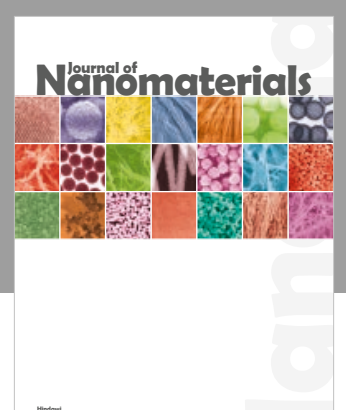

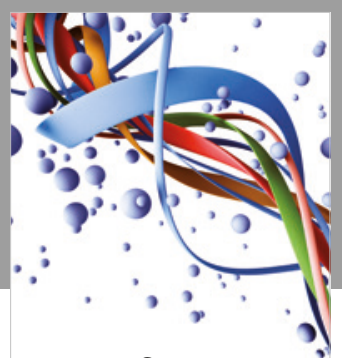

Scientifica

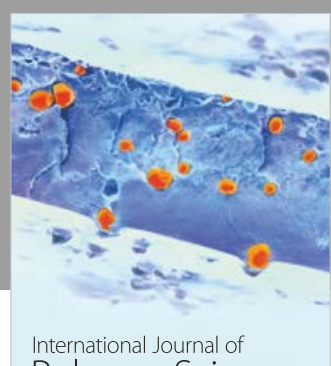

Polymer Science

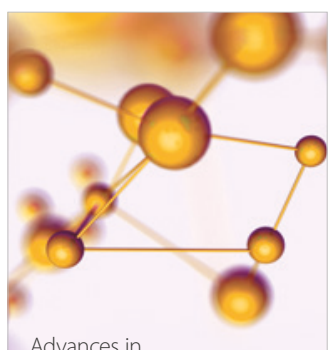

Physical Chemistry
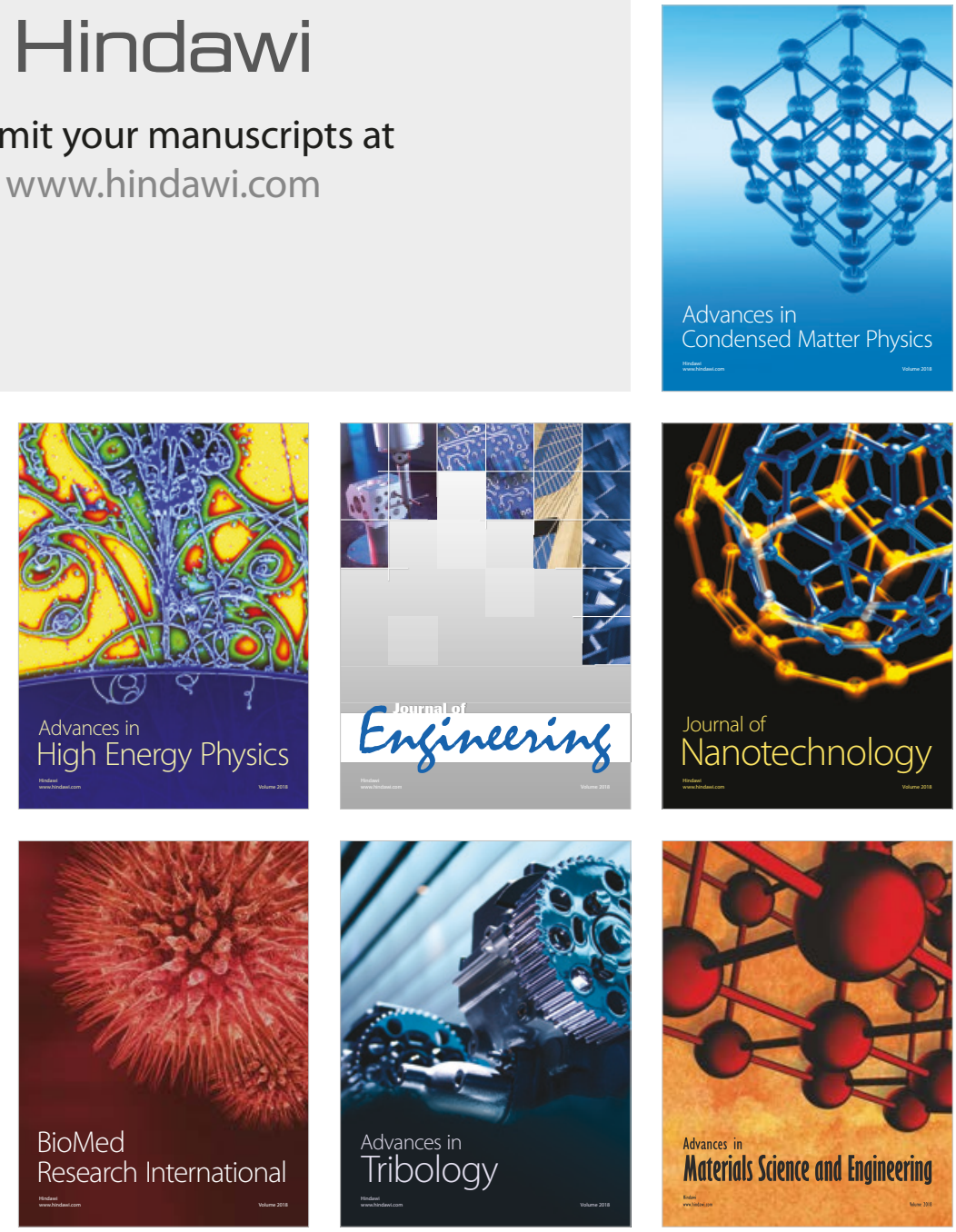